\title{
Strategies for children's hospital in response to COVID-19 pandemic: perspective and practice at a designated pediatric hospital in Shanghai, China
}

\author{
Xiao-Bo Zhang ${ }^{1}$ - Xiao-Jing Hu ${ }^{1}$ Xiao-Wen Zhai ${ }^{1}$. Ying Gu ${ }^{1}$. Lin Yuan ${ }^{1}$. Peng Shi ${ }^{1}$ Xiao-Lin Ge ${ }^{1}$. Gong-Bao Liu ${ }^{1}$. \\ Guo-Mei Shen ${ }^{1}$. Chuan-Qing Wang ${ }^{1}$ - Ai-Mei Xia ${ }^{1}$ - Mei Zeng ${ }^{1}$ - Guo-Ying Huang ${ }^{1,2}$
}

Received: 3 May 2020 / Accepted: 18 September 2020 / Published online: 15 October 2020

(c) Children's Hospital, Zhejiang University School of Medicine 2020

The coronavirus disease 2019 (COVID-19) caused by severe acute respiratory syndrome coronavirus-2 (SARS-CoV-2) has become a global pandemic [1-5]. As of July 31, 2020, there were 17,106,007 infected patients and 668,910 deaths worldwide [6, 7]. National protocols regarding COVID-19 issued by national-level agencies need to be revised in the field of pediatrics [8]. From the perspective of hospital management, identification and set-up of isolation wards and clinics, modification of workflow and processes, management of staff, allocation of medical resources, and formulation of clinical guidelines and protocols in infection prevention and control (IPC) were addressed as response measures to reduce the risk of transmission to healthcare workers and patients [9]. In this paper we describe the strategies and practices implemented at the Children's Hospital of Fudan University in response to this public health crisis. The strategy of response measures is shown in Supplementary Fig. 1.

\section{Management of outpatient and emergency departments}

\section{Triage protocol}

Medical staff in outpatient and emergency departments were equipped with different levels of personal protective equipment (PPE) as stratified by risk, including protective clothing, mask

Electronic supplementary material The online version of this article (https://doi.org/10.1007/s12519-020-00394-w) contains supplementary material, which is available to authorized users.

Guo-Ying Huang

gyhuang@shmu.edu.cn

1 Children's Hospital of Fudan University, 399 Wan Yuan Road, Shanghai 201102, China

2 Shanghai Key Laboratory of Birth Defects, Shanghai, China or respirator, goggles or face shield, and gloves, among other equipment [10-12]. Dedicated entrances and exits in the outpatient and emergency departments were clearly marked. Other entrances and pathways were closed. Thermal imaging cameras were used to screen the body temperature of patients, family members and visitors. Family members of patients and visitors were required to complete an epidemiological history checklist on a phone app before visiting the hospital (Supplementary Table 1). Upon arrival at the hospital, children had their temperature assessed in addition to having their parent complete an epidemiologic surgery before entry. There were measures taken to ensure that waiting areas were well ventilated and social distancing of at least one meter was enforced. The circulating nurses re-measured the body temperature of the children as a secondary pre-check. During consultation in the clinic room, a standardized clinical and epidemiological history assessment was confirmed by medical staff. Suspected cases were identified through criteria including body temperature above $37.3^{\circ} \mathrm{C}$, high-risk epidemiological history, or respiratory symptoms. These patients were transferred to the COVID-19 clinic for further diagnosis and medical observation. In the COVID-19 clinic, medical staff were provided the highest necessary level of IPC, comprehensive PPE including hat, N95 respirator, face shield, goggles, gloves, coverall protective clothing, and boot covers [10-12]; medical staff sought advice from the hospital expert group for risk assessment in a timely manner. A decision whether to admit a patient was decided by the expert group, followed by medical staff from the COVID-19 clinic escorting these children through a designated corridor.

\section{Management of hospitalized children}

\section{Admission guidance and management}

Upon admission, children and their caregivers were educated on the knowledge of COVID-19 infection prevention, hand 
hygiene, and proper wearing of face masks. Designated caregivers were asked to wear masks during the child's admission. The movement of caregivers in the ward was restricted, with electronically controlled access to the ward. The body temperatures of visitors were measured at the entrance of the ward with their contact history closely monitored and verified by electronic recordkeeping. Caregivers should take the highest level of precautions and were required to wear proper PPE, including protective clothing, goggles, surgical mask, and gloves.

\section{Transition room management}

Transition rooms were established for children from regions outside of Shanghai to be under medical observation for at least 3 days. Risk assessment was carried out daily by medical and nursing staff according to contact history and clinical manifestations to decide whether transfer to the regular rooms is warranted. Activities and movement of patients and their caregiver were restricted in the transition rooms. Medical staff should ensure adequate use of PPE when entering the transition rooms.

\section{COVID-19 ward management}

The COVID-19 ward was the dedicated quarantine ward established in the infectious disease building of the hospital campus. The ward was divided into three areas, including clean, semi-dirty, and dirty areas. The AIIR was managed in a closed system, with minimum 12 air changes per hour and negative pressure monitoring is no less than -15 pa [11]. PPE was required to be fitted for medical staff when they enter the AIIR. A comprehensive powered ventilation respirator mask was required for collecting specimens or performing invasive procedures for effective protection. Visitors were prohibited from entering the AIIR, and video calls were offered as an alternative for social contact and to ensure emotional well-being. Recreational and play activities for children were carried out while maximizing use of technology such as internet-connected devices. Each patient's clinical condition was monitored closely, and when carrying out procedures, protective measures were required to be strictly adhered to. It was imperative that the airway was managed in a closed system.

\section{Management of discharged children}

Individualized health education was provided to all inpatients prior to discharge. Education of families with emphasis on disinfection of the home environment, hand hygiene, disinfection and management of feces, and proper coughing and sneezing etiquette. Proper follow-up of the clinical condition of discharged children was done through the mobile phone app, and measures were taken to ensure that families were proficient in use of the app. COVID-19 positive patients were required to continue in-home isolation for at least 2 weeks, and follow-up at the COVID-19 clinic after 2 weeks and 4 weeks [13].

\section{Management of computed tomography scan and laboratory test}

\section{Computed tomography examination process}

Confirmed or suspected COVID-19 patients were transferred in negative pressure isolators to the dedicated computed tomography (CT) room within the infectious disease building. The medical staff in the COVID-19 clinic or quarantine ward notified the radiology department and submitted a CT request. Doctors, nurses, technicians, and workers with close contact with the patients were all required to use the highest level of PPE (grade III protection, with airtight respirator, protective clothing, goggles, and gloves) during the process of transfer After the CT scan was completed for confirmed or suspected COVID-19 patients, technicians wearing PPE disinfected the CT room, buffer zone, equipment surfaces, and the air in the room was left to settle for 1 hour. For patients without COVID-19, the room was thoroughly disinfected three times a day.

\section{Laboratory test process}

For confirmed or suspected COVID-19 patients, one oropharyngeal swab and one nasopharyngeal swab were collected with comprehensive powered ventilation respirator mask for effective protection. Only synthetic fiber swabs with plastic handles were used to collect specimens. The specimens were kept in the tubes containing $3 \mathrm{~mL}$ virus preservation solution. All specimens collected had to be correctly labeled with each patient's identifying information and double bagged with two sealable specimen bags, transported with an ice pack in a delivery box monitored to be between 2 and $8{ }^{\circ} \mathrm{C}$.

\section{Management of support systems}

\section{Guideline issue and update}

With the evolving situation during the outbreak, the hospital guidelines were modified frequently according to the latest guidelines issued by health authorities from the National Health Commission of the People's Republic of China, 
Shanghai Pediatric Clinical Quality Control Center, and local experts' opinions [14].

\section{Staff management}

Hospital staff were centrally managed by the department of human resources. Staff whereabouts were managed with meticulous tracing of staff epidemiologic history. Mass gatherings were canceled. Staff were required to quarantine at home if they had returned from areas outside of Shanghai. Staff allocation was adjusted to the work demand of IPC and patients by the Medical Affairs and Nursing Departments. Health professionals were redeployed to support the work on the COVID-19 clinic, the quarantine ward, and the front-line in outpatient department and inpatient wards. Contingency work shifts with limited number of doctors and nurses were arranged in case of mass isolation and critical care of patients. Both medical and non-medical personnel were trained for proper use of PPE, collection and transport of specimens, and disinfection of the clinical environment [11, 12]. Continuous training was provided to staff for updated knowledge of clinical diagnosis and treatment of COVID-19 and IPC.

\section{Infection prevention and control training}

A 12-minute training video was developed on essential infection protection and control measures. The video put a strong emphasis on the correct use of PPE especially the doffing part which has been documented to be a high-risk procedure leading to disease transmission (https://mp.weixi n.qq.com/s/zhYFlHJNQ3euFNmWRneY2A). The whole process of donning and doffing is done under supervision (Supplementary Figs. 2 and 3) [15, 16]. Masks were required to be replaced every 4 hours.

\section{Personal protective equipment management}

Increased supplies of PPE were sourced from various channels, including manufacturers, community donations, and distribution from government agencies. PPE was prioritized to be allocated first to the front-line healthcare workers fitted with the differential level of PPE according to risk. N95 respirator masks were used only for staff working at the COVID-19 clinic and in the suspected/confirmed patient ward [level I (+4), level II and level III], while surgical masks were used for staff working in the general medical area and semi-contaminated areas [from level I (1) to level I (+3)] [14]. There was strict monitoring of the number of patients and PPE capacity in the hospital. With the decrease of routine medical service for outpatient and inpatient and increasing demand for suspected/confirmed COVID-19 cases, the number of each type of PPE remaining in stock was recorded every day. The consumption of each type of PPE per day and the average consumption of PPE per day and per staff were calculated.

\section{Important aspects to ensure patient and staff safety}

There was no nosocomial COVID-19 infection among patient or staff during the outbreak and pandemic periods at the hospital. We provided adequate PPE and training courses to prevent nosocomial infections between patients and staff. The number of patients and capacity of support systems from February to July are shown in Supplementary Tables 2 and 3, totally a cost of about 449,028 RMB (about 65,551 USD). There were 568 doctors and 537 nurses in service in February, 485 doctors and 562 nurses in service in March, of whom none got infected with COVID-19. As of July 31, 2020, sixty-one patients with confirmed COVID-19 and 92 suspected COVID-19 patients were treated at our hospital. There were no critical patients at our hospital.

Our experience shows that it is practical improve vigilance, initiate early management of patient access, triage, and to provide follow-up. It is also useful to conduct strict surveys of epidemiological history, to supervise medical staff, and adhere to restrictions on mass gatherings and movement. In the COVID-19 isolation room, it is essential to implement comprehensive high-level infection control and prevention measures, as well as to prevent hospital infections for the protection of staff and vulnerable patients. For the COVID-19 ward, because admitted children need to be temporarily separated from their parents during the isolation period, nurses acted as caregivers and were required to accompany children at all times. Nurses were required to use fully protective powered air supply filter respirators to ensure the success of procedures and that they are fully protected when carrying out invasive procedures, such as venepuncture, other puncture procedures, and suctioning. In addition to protecting the health of children and medical staff, we also actively engaged with staff by means of making available a mental health consultation hotline for staff to reduce stress and anxiety during the outbreak. Our hospital optimized the supporting resources of personnel, beds, and PPE consumption to meet the demands of health services.

The COVID-19 pandemic may persist for a long period of time, during this time, prevention and control measures must be strictly adhered to in children's hospitals. Examples of these measures include social distancing, hand hygiene, and strict compliance with hospital strategies, which can effectively prevent the virus spread. In the post-pandemic era, the information systems used for contact tracing and technologies used for this, in addition to temperature monitoring, 
mask wearing, and social distancing have become routine procedures in our hospital.

In conclusion, we are still in the struggle against the COVID-19 and other infectious disease pandemic with other countries and regions. The strategies and practices of response measures in our hospital have been effective and should be shared with other medical facilities, especially children's hospitals.

Acknowledgements The authors would like to acknowledge Conway Niu, Ph.D. from Aberdeen Maternity Hospital, Aberdeen, Scotland for the help in preparing the manuscript.

Author contributions HGY conceptualized and designed the study, supervised all analyses, critically reviewed and revised the manuscript. ZXB, HXJ and ZXW designed the study, coordinated and supervised data collection, conducted the data analyses, drafted and revised the manuscript. ZXB, HXJ and ZXW contributed equally to this work. GY, YL, SGM and XAM designed the data collection instruments, collected data, carried out the initial analyses. LGB assisted in data clearing, interpretation of the results and revised the manuscript. SP and GXL critically reviewed the manuscript for study design and statistical analysis. WCQ and ZM critically reviewed the manuscript for important intellectual content. All authors approved the final manuscript as submitted and agreed to be accountable for all aspects of the work.

Funding None.

\section{Compliance with ethical standards}

Ethical approval Not needed.

Conflict of interest The authors declared no financial conflict of interest.

\section{References}

1. World Health Organization. Coronavirus disease (COVID-2019) situation reports. 2020. https://www.who.int/docs/default-sourc e/coronaviruse/situation-reports/20200310-sitrep-50-covid-19. pdf?sfvrsn=55e904fb_2. Accessed 10 Mar 2020.

2. Zou LR, Ruan F, Huang MX, Liang LJ, Huang HT, Hong ZS, et al. SARS-CoV-2 viral load in upper respiratory specimens of infected patients. N Engl J Med. 2020;382:1177-9.

3. Chen NS, Zhou M, Dong X, Qu JM, Gong FY, Han Y, et al. Epidemiological and clinical characteristics of 99 cases of 2019 novel coronavirus pneumonia in Wuhan, China: a descriptive study. Lancet. 2020;395:507-13.
4. Zhu N, Zhang DY, Wang WL, Li XW, Yang B, Song JD, et al. A novel coronavirus from patients with pneumonia in China, 2019. N Engl J Med. 2020;382:727-33.

5. World Health Organization. WHO Director-General's opening remarks at the media briefing on COVID-19-11 March 2020. https://www.who.int/dg/speeches/detail/who-director-general-sopening-remarks-at-the-media-briefing-on-covid-19---11-march -2020. Accessed 11 Mar 2020.

6. World Health Organization.Novel coronavirus (COVID-19) situation. 2020. https://www.who.int/emergencies/diseases/novel -coronavirus-2019. Accessed 11 Mar 2020.

7. Centers for Disease Control and Prevention. Locations with confirmed COVID-19 cases global map. 2020. https://www.cdc.gov/ coronavirus/2019-ncov/locations-confirmed-cases.html. Accessed 11 Mar 2020.

8. Zeng LK, Tao XW, Yuan WH, Wang J, Liu X, Liu ZS, et al. First novel coronavirus pneumonia in newborn in China. Chin J Pediatr. 2020;58:E009 (in Chinese).

9. Wong J, Goh QY, Tan Z, Lie SA, Tay YC, Ng SY, et al. Preparing for a COVID-19 pandemic: a review of operating room outbreak response measures in a large tertiary hospital in Singapore. Can J Anaesth. 2020;67:732-45.

10. Centers for Disease Control and Prevention.Infection prevention and control recommendations for hospitalized patients under investigation (PUIs) for Ebola Virus Disease (EVD) in U.S. Hospitals. 2020. https://www.cdc.gov/vhf/ebola/clinicians/evd/infec tion-control.html. Accessed 11 Mar 2020.

11. Centers for Disease Control and Prevention. Isolation precautions. 2020. https://www.cdc.gov/infectioncontrol/guidelines/isolation/ index.html. Accessed 11 Mar 2020.

12. Yang TL, Xia AM, Hu XJ, Yu LZ, Shi H, Gu Y, et al. Novel coronavirus pneumonia in children: personal protection and specimen collection methods. Chin J Evid Based Pediatr. 2020;15:19-211 (in Chinese).

13. National Health Commission. Diagnosis and treatment plan on 2019 novel coronavirus disease (version 7). 2020. https://www. nhc.gov.cn/yzygj/s7653p/202003/46c9294a7dfe4cef80dc7f591 2eb1989.shtml. Accessed 3 Mar 2020.

14. Zhang XB, Wang CQ, Zhu QR, Wang Y, Xu H, Huang GY, et al. Guiding opinions on the quality control of pediatrics in Shanghai about the prevention and control of COVID-19 (Version 1.0). Fudan Univ J Med Sci. 2020;47:161-9.

15. Centers for Disease Control and Prevention. Interim guidance for persons who may have 2019 Novel Coronavirus (2019-nCoV) to prevent spread in homes and residential communities. 2020. https ://www.cdc.gov/coronavirus/index.html. Accessed 11 Mar 2020.

16. National Children's Medical Center. Donning and Doffing of Personal Protective Equipment when in contact with COVID-19 patients. 2020. https://mp.weixin.qq.com/s/zhYFlHJNQ3euFNm WRneY2A. Accessed 11 Mar 2020.

Publisher's Note Springer Nature remains neutral with regard to jurisdictional claims in published maps and institutional affiliations. 